\title{
Before the Bow Breaks: Tradition vs. Nutrition for Cuba's Next Generation
}

\section{By Lázaro Alfonso, MD, MS}

Changing dietary habits for most adults requires a will of steel. After all, our preferences were acquired decades ago: while we learned to crawl, talk and walk, we also learned to eat. Yet, "acquire" is probably the wrong verb. "Instill" comes closer to describing the way parents and other caregivers pass down the culture of food from one generation to another in all societies.

In Cuba, this is turning out to be the crux of our nutritional dilemma today. Part of our problems stem from the Spanish influence in our diet, explaining our penchant for pork and its myriad derivatives, along with animal fats and milk products. Then there is our history of indiscriminate and over-consumption of refined sugar in almost every form, reinforced by more than a century of monoculture. And to this we add the traditional popular view that vegetables just don't taste good, and some aren't even nutritious. Salad is decorative, but certainly not a core part of the usual Cuban meal.

Such dietary prejudices figure strongly into parents' decisions about what to feed their infant or toddler. Their choices not only influence future tastes in food-they are determining factors in the child's growth and development at this all-important stage that requires a balance between foods that provide energy and those that regulate bodily functions. My experience as a pediatrician and nutritionist leads me to believe that these inherited cultural patterns are one of the most difficult obstacles we face to attaining adequate nutrition for our children, the next generation of $\mathrm{Cu}$ bans. This is particularly disturbing as more Cuban children today are overweight and obese. According to the 2nd National Survey on Risk Factors and Chronic Diseases, by $2001,13 \%$ of children aged 1-14 years were overweight or obese and therefore more likely to grow into overweight, obese and unhealthy adults.

We have made some progress: all Cuban maternity hospitals have been accredited by UNICEF as Mother and Baby Friendly Hospitals, with nearly $99 \%$ of mothers breastfeeding upon discharge (over $99 \%$ of births in Cuba take place in hospitals). Breastfeeding, however, begins to decline once mother and baby return home: just $41 \%$ of infants are exclusively breastfed during the first six months of life.

Moreover, it's when solid foods are introduced-a definitive stage in developing dietary habits-that the bigger controversies often begin between pediatricians and caregivers. While adhering to rigid and often obsolete rules makes no sense, letting folk tales (masking as folk wisdom) overrule science is equally misguided. (An old Cuban saying about squash goes like this: "You can rub it on or swallow it, the result is the same...nil.") We hear from parents who are guided by great-grandmother's admonitions that potatoes cause heartburn, eggs are bad for the liver, and vegetables cause indigestion. If all this were true, we could only recommend potatoes when a child turns five, prescribe eggs in fortified drinks only for sick adults, and rarely recommend carrots to anyone.
This logic steeped in tradition also appears on other fronts, such as the tendency among some parents to introduce solid foods too early or to "supplement" breastfeeding with sugar-laden cow's milk ("the child isn't getting enough to eat!"); over-protection and overindulgence by parents, grandparents, and others who offer sweets as rewards or as tranquilizers; forcing food on toddlers, unappreciative of their tastes; and organizing feeding times and meals chaotically or only at the family's convenience. Not to mention the prevailing beauty standards that regard a chubby child a healthy one.

\section{...inherited cultural patterns are one of the most difficult obstacles we face to attaining adequate nutrition for our children.}

The end result is that pediatricians dedicated to clinical nutrition are overwhelmed with patients brought to us when the damage has already been done-infants and toddlers suffering from inadequate management of complementary solid foods, insufficient weight gain, or more frequently now, tendencies towards excessive weight gain and obesity. Where I practice, we often see small children with clinical signs of obesity, brought in by obviously overweight parents. Sometimes we ask the brothers, sisters, and grandparents to come along, only to find that they are all overweight. Granted the existence of genetic predisposition, in my experience the family's dietary culture is the more obvious problem.

\section{How, then, can we turn this tide?}

I am convinced that more public education-health promotion-must be at the heart of our efforts, and we must generate strategies that take advantage of the strengths of our universal health care system. We have a broad network of primary care facilities with doctors, nurses, social workers, and psychologists at the community level. There are child care centers that cater to families of working mothers, staffed by educators, nurses and even physicians. We have publicly-owned mass media. A healthy pregnant woman in Cuba sees a doctor 8-10 times before giving birth, and high-risk women have many more doctor visits. We have Mother and Baby Friendly Hospitals, responsible parenthood classes, and a well-educated population.

In order to harness these resources, we need to include more training on nutritional issues for all our health care providers so that they can provide effective leadership and counseling wherever they are in the health system. This effort should begin in our medical schools, where clinical nutrition is not yet included in the core curriculum. We hope this will be accomplished soon.

Like countries around the world, we have no choice but to change our dietary habits: the epidemic noncommunicable chronic conditions that threaten health worldwide-obesity, diabetes, hypertension, atherosclerosis, dislipidemias, and others-have their point of departure in early infancy. And by the time this generation of toddlers grows into adulthood, it will be too late to change-both for them and for health systems bowed by such an out-of-control disease burden. $-\mathrm{p}$ - 